\title{
ANÁLISE COMPARATIVA DA COMPOSIÇÃO QUÍMICA VOLÁTIL DE AMOSTRAS DO ALECRIM IN NATURA COM A DISPONÍVEL COMERCIALMENTE
}

\author{
A. L. M. CAMELO*, F. C. OLIVEIRA, F. F. M. SILVA, F. T. C. SOUZA e E. LONGHINOTTI \\ Programa de Pós-Graduação em Química - Universidade Federal do Ceará \\ andreluiz0502@yahoo.com.br
}

Artigo submetido em abril/2014 e aceito em outubro/2014

DOI: $10.15628 /$ holos.2014.2082

\section{RESUMO}

As plantas medicinais têm ampla gama de aplicações, devido às suas potenciais atividades biológicas, com um ou mais princípios ativos relevantes para a saúde humana. Este fato torna-se preocupante a partir do momento que há um aumento crescente na comercialização das mesmas, pois, determinados tipos de ervas, de composições químicas e propriedades farmacológica diferentes são muitas vezes comercializados com nomes populares idênticos, como é o caso do alecrim, que designa várias espécies de plantas medicinais. Portanto, este estudo teve como objetivo realizar a extração e análise comparativa da composição química do óleo essencial de uma amostra comercial, que não descreve sua identificação botânica e uma amostra in natura, sendo ambas reconhecidas pelo nome de alecrim. Foram observadas diferenças significativas entre a composição química das amostras, confirmando a importância de um maior detalhamento quanto a sua classificação botânica e constituição química na descrição das plantas medicinais comercializadas e utilizadas pela população, a fim de evitar a utilização indevida desta fonte natural, uma vez que as propriedades farmacológicas atribuidas a cada espécie estão intimimamente relacionada com a sua composição química.

\section{COMPARATIVE ANALYSIS OF THE CHEMICAL COMPOSITION OF VOLATILE SAMPLES OF ROSEMARY IN NATURE WITH COMMERCIALLY AVAILABLE}

\begin{abstract}
Medicinal plants have a wide range of applications due to their potential biological activities, with many active principles that are relevant to human health. This is a fact that has been becoming of concern due to the increasing in the commercialization of such plants, since certain types of herbs, of different chemical compositions and pharmacological properties are often marketed with identical popular names. We can cite rosemary, which designate several species of medicinal plants. Therefore, this study aimed to perform an extraction followed by a comparative analysis between the chemical composition of the essential oil present in a commercial sample (with
\end{abstract}

a non-specified botanical identification) and a sample in nature, both recognized as being rosemary. Significant differences between the chemical composition of the samples were observed, confirming the significance of providing further details as to its botanical classification and chemical constitution, when describing medicinal plants commercialized and used by the population. This is especially important to avoid misuse of this natural source, since the pharmacological properties attributed to each species are closely related to its chemical composition. 


\section{INTRODUÇÃO}

As plantas medicinais possuem largo espectro de aplicações devido as suas potenciais atividades biológicas, apresentando um ou mais princípios ativos, relevantes para à saúde humana (FERREIRA et al., 1998). A recorrência a essas plantas como recurso terapêutico ocorre desde tempos imemoriais, tendo sofrido a influência de diversas culturas, com seus mitos, credos e sabedorias (HOUGHTON, 1995).

Muitas espécies de plantas encontradas no Nordeste brasileiro apresentam ampla distribuição geográfica e boa adaptação ao clima do semi-árido (LEAL-CARDOSO; FONTELES, 1999). O estudo das plantas odoríferas do Nordeste resultou na descoberta de vários óleos essenciais de elevado potencial econômico, sendo alguns deles fonte importante de matérias-primas para a indústria química enquanto outros são dotados de propriedades aromatizantes, farmacológicas, terapêuticas e anti-sépticas (CRAVEIRO, 1981).

As diversas atividades atribuídas aos óleos essenciais estão diretamente relacionadas com a sua composição química, sendo esta, em geral, bastante complexa em cada espécie de planta e observando-se larga variação até mesmo em espécies cujos nomes populares coincidem. Este fato tem chamado a atenção, nas últimas décadas, em que se observa uma crescente comercialização destes produtos para uso medicinal (CALIXTO, 2000; SUZUKI, 2002; NUNES, 2003; DAMIANI et al., 2014), sem a devida especificação da identidade botânica da planta, podendo assim, resultar em uso inadequado e riscos para os consumidores.

Nesse contexto, o alecrim é um nome popular utilizado para diversas espécies de plantas aromáticas medicinais, muito embora estas possuam composições químicas voláteis diferentes e consequentemente propriedades farmacológicas distintas.

Dentre estas merece destaque, a Lippia sidoides, por apresentar várias propriedades farmacológicas e largo uso popular. Conhecida popularmente por alecrim, alecrim-pimenta, alecrim do nordeste e alecrim-bravo, sendo suas folhas utilizadas para tratamento de acne, ferimentos, infecções da pele e do couro cabeludo. Na forma de infusão tem sido usada para rinite alérgica e no tratamento das infecções, vaginais, da boca e da garganta (MATOS, 2007). O óleo essencial de Lippia sidoides possui reconhecida atividade antimicrobiana (BERTINI et al., 2005; FONTENELLE et al., 2007; VERAS et al., 2012; OLIVEIRA ET al., 2014).

Além da Lippia sidoides, outras espécies são comercializadas com o nome alecrim, tais como: Holocalyx glaziovii Taub. ex Glaz, Lantana microphylla Franch, Rosmarinus officinalis L., Dicliptera aromatica L., sendo relatada propriedades terapêuticas diversas, como: antiespasmódico, desordens respiratórias, analgésicos, anti-reumática, diurético dentre outras (ALSEREITIA et al., 1999).

Este trabalho teve como objetivo realizar a extração e a análise do óleo essencial de uma amostra comercial especificada com o nome de alecrim (com espécie botânica não discriminada) e de um alecrim in natura, visando analisar possíveis diferenças entre a composição química das mesmas e com isso contribuir com subsídios técnicos que fomentem uma política de qualificação na comercialização de plantas medicinais, incorporando a estes produtos as especificações botânicas e químicas pertinentes. 


\section{MATERIAIS E MÉTODOS}

\subsection{Material Botânico}

As folhas frescas de $L$. sidoides foram coletadas no Horto de Plantas Medicinais Francisco José de Abreu Matos, na Universidade Federal do Ceará (UFC) no dia 14 de janeiro de 2013. As folhas desidratadas da amostra comercial designada por Alecrim foram obtidas no comércio local de Fortaleza-CE.

\subsection{Obtenção dos óleos essenciais de L. sidoides e do Alecrim comercial}

A extração do óleo essencial foi realizada em um sistema tipo Clevenger. Para isso, $100 \mathrm{~g}$ das folhas frescas de $L$. sidoides e $100 \mathrm{~g}$ de folhas desidratada do Alecrim comercial foram pesados, adicionadas separadamente em balões de fundo redondo com capacidade de 2,0 litros e foi utilizado um volume de água destilada suficiente, para submergir o material vegetal. Este procedimento foi realizado em triplicata. O tempo de extração do óleo essencial foi de 2,0 horas, o qual foi separado, seco com sulfato de sódio anidro $\left(\mathrm{Na}_{2} \mathrm{SO}_{4}\right)$, filtrado e armazenado em freezer ao abrigo da luz.

\subsection{Análise dos voláteis por CG-EM e CG-DIC.}

Após o processo de extração, os óleos essenciais foram analisados, simultaneamente por um Cromatógrafo Gasoso Acoplado ao Espectrômetro de Massa (CG-EM) e um Cromatógrafo Gasoso com Detector de Ionização por Chama (CG-DIC). Para isso, preparou-se uma solução contendo $2 \mu \mathrm{L}$ de óleo essencial em $1 \mathrm{~mL}$ de diclorometano. As análises foram realizadas em um CG-EM da marca Shimadzu (modelo GCMS - QP 2010) utilizando uma coluna capilar DB-5, fase estacionária $5 \%$ difenildimetilsiloxano de 20 metros de comprimento, diâmetro interno de 0,18 $\mathrm{mm}$ e com espessura de filme de $0,40 \mu \mathrm{m}$ (J\&W Scientific ${ }^{\circ}$ ). A programação de aquecimento do forno cromatográfico estabelecida foi de $40{ }^{\circ} \mathrm{C}$ ( 3 minutos) $\rightarrow 3^{\circ} \mathrm{C} /$ minuto até $290^{\circ} \mathrm{C}$ (4 minutos). Os parâmetros instrumentais utilizados foram: temperatura do injetor de $250^{\circ} \mathrm{C}$; modo de injeção split de 1:10; vazão volumétrica da fase móvel de 0,59 mL/minuto; temperatura da interface de $300^{\circ} \mathrm{C}$; análises realizadas no modo SCAN em uma faixa de 18 a $400 \mathrm{~m} / \mathrm{z}$ (em intervalos de $0,5 \mathrm{~s} \mathrm{e}$ com energia de ionização de $70 \mathrm{eV}$ ); temperatura do detector de $250{ }^{\circ} \mathrm{C}$. Já para a análise quantitativa foi utilizado o CG-DIC da marca Shimadzu (modelo GC-2010) utilizando uma coluna capilar Rtx-5, fase estacionária 5\% difenildimetilsiloxano, de 30 metros de comprimento, diâmetro interno de 0,25 $\mathrm{mm}$ e espessura do filme de $0,25 \mu \mathrm{m}$ (Restek ${ }^{\circledR}$ ). Para as análises a programação de aquecimento do forno cromatográfico estabelecida foi de $40{ }^{\circ} \mathrm{C}$ ( 3 minutos) $\rightarrow 3^{\circ} \mathrm{C} /$ minuto até 290 ${ }^{\circ} \mathrm{C}$ (4 minutos). Os parâmetros instrumentais foram: temperatura do injetor de $250{ }^{\circ} \mathrm{C}$; modo de injeção split de 1:5; vazão volumétrica da fase móvel de $0,59 \mathrm{~mL} /$ minuto e temperatura do detector de $220^{\circ} \mathrm{C}$. Para todas as análises utilizou-se o programa GC-MS Solution e GC Solution - versão 2.30 (Shimadzu ${ }^{\circledR}$ ), respectivamente, para obtenção e tratamento dos dados cromatográficos.

2.4 Análise qualitativa dos constituintes voláteis dos óleos essenciais obtidos 
Os constituintes voláteis foram identificados pela comparação dos espectros de massas obtidos, com os espectros de massas do banco de dados do equipamento (NIRST 05, NIRST 27, WILEY 229). Neste caso, o índice de similaridade entre os espectros foi utilizado para avaliar e identificar os compostos na amostra. Além disso, utilizou-se o Índice de Retenção Linear (IRL) como parâmetro de identificação dos compostos presentes na amostra (Viegas e Bassoli, 2007). Para isto, uma solução de $100 \mu \mathrm{g} / \mathrm{mL}$ de uma série homóloga de hidrocarbonetos de C-7 a C-30 foi preparada para ser calculado o IRL. Sendo assim, $1 \mu \mathrm{L}$ dessa solução foi injetada, nas mesmas condições instrumentais de análise descritos anteriormente para CG-EM e CG-DIC. O IRL de cada composto foi calculado através da Equação 1 e comparado com valores da literatura (ADAMS, 2001; Banco de dados PHEROBASE).

$$
\mathbf{I R L}=\mathbf{1 0 0}\left[\mathbf{n}+(\mathbf{N}-\mathbf{n}) \frac{\mathbf{t}_{\mathbf{R}}^{\prime}(\text { Desconhecido })-\mathbf{t}_{\mathbf{R}}^{\prime}(\mathrm{n})}{\mathbf{t}_{\mathbf{R}}^{\prime}(\mathrm{N})-\mathbf{t}_{\mathbf{R}}^{\prime}(\mathrm{n})}\right]
$$

Equação (1)

Em que, $\mathrm{n}$ é o número de átomos de carbono no menor alcano; $\mathrm{N}$ é o número de átomos de carbono no maior alcano; $t^{\prime}{ }_{R}(n)$ é o tempo de retenção ajustado no menor alcano; $t^{\prime}{ }_{R}(N)$ é o tempo de retenção ajustado no maior alcano. A análise quantitativa de cada componente foi determinada pelo método da normalização.

\section{RESULTADOS E DISCUSSÕES}

Após as análises dos constituintes de cada óleo essencial foi possível identificar um total de 24 componentes para a L. sidoides e 27 componentes para o alecrim comercial de acordo com o descrito na Tabela 1.

Tabela 1. Composição Química dos óleos essenciais da amostra de Alecrim in natura (Lippia sidoides) e da amostra de Alecrim comercial.

\begin{tabular}{|c|c|c|c|c|c|c|}
\hline \multirow[b]{2}{*}{ № } & \multirow[b]{2}{*}{ T.R } & \multirow[b]{2}{*}{ Composto } & \multirow[b]{2}{*}{ IR calc. } & \multirow[b]{2}{*}{$\mathbf{I R}_{\text {Lite. }}$} & \multicolumn{2}{|c|}{ Normalização } \\
\hline & & & & & in natura & Comercial \\
\hline 1 & 13,27 & $\alpha-$ Tujeno & 926 & 924 & 1,11 & 0,07 \\
\hline 2 & 13,70 & $\alpha-$ Pineno & 933 & 932 & 0,27 & 9,72 \\
\hline 3 & 14,50 & Canfeno & 948 & 946 & ND & 3,15 \\
\hline 4 & 15,75 & Sabineno & 973 & 969 & 1,12 & ND \\
\hline 5 & 15,88 & $\beta$-Pineno & 976 & 974 & 0,08 & 1,58 \\
\hline 6 & 16,69 & Mirceno & 992 & 988 & 1,80 & 0,99 \\
\hline 7 & 17,33 & $\alpha-$ Felandreno & 1005 & 1002 & 0,17 & 0,09 \\
\hline 8 & 17,65 & $3-\delta-$ Careno & 1010 & 1008 & 0,14 & 0,28 \\
\hline 9 & 17,98 & $\alpha$-Terpineno & 1017 & 1014 & 2,17 & ND \\
\hline 10 & 18,39 & $\rho-$ Cimeno & 1025 & 1022 & 18,96 & ND \\
\hline 11 & 18,48 & o-Cimeno & 1025 & 1020 & ND & 1,84 \\
\hline 12 & 18,62 & Limoneno & 1029 & 1024 & 0,28 & ND \\
\hline 13 & 18,73 & Eucaliptol & 1031 & 1033 & 1,01 & 49,14 \\
\hline 14 & 19,12 & $Z-\beta$-Ocimeno & 1038 & 1037 & 0,03 & ND \\
\hline 15 & 19,20 & $E-\beta$-Ocimeno & 1039 & 1044 & 0,15 & ND \\
\hline 16 & 20,19 & $\nu$-Terpineno & 1059 & 1054 & 10,25 & 0,16 \\
\hline 17 & 20,59 & Cis -Sabineno hidratado & 1067 & 1065 & 0,19 & ND \\
\hline 18 & 21,70 & Terpinen - 4-ol & 1177 & 1174 & 0,06 & ND \\
\hline 19 & 21,80 & Terpinoleno & 1090 & 1086 & ND & 0,15 \\
\hline 20 & 22,41 & Linalol & 1102 & 1096 & ND & 1,19 \\
\hline 21 & 23,14 & Endo - Fechol & 1116 & 1114 & ND & 0,08 \\
\hline
\end{tabular}




\begin{tabular}{|c|c|c|c|c|c|c|}
\hline 22 & 23,67 & $\alpha-$ Canfolenal & 1127 & 1122 & ND & 0,05 \\
\hline 23 & 24,41 & Trans - Pinocarveol & 1142 & 1135 & ND & 0,07 \\
\hline 24 & 24,62 & Cânfora & 1146 & 1141 & ND & 19,27 \\
\hline 25 & 24,87 & Canfeno Hidratado & 1151 & 1145 & ND & 0,05 \\
\hline 26 & 25,26 & Isopulegol & 1159 & 1145 & ND & 0,05 \\
\hline 27 & 25,40 & Cis - Pinocanfono & 1162 & 1172 & ND & 0,04 \\
\hline 28 & 25,77 & Borneol & 1170 & 1165 & ND & 4,98 \\
\hline 29 & 26,69 & Cimen -8 -ol & 1188 & 1179 & ND & 0,07 \\
\hline 30 & 26,99 & $\alpha-$ Terpineol & 1194 & 1186 & ND & 4,10 \\
\hline 31 & 27,27 & Anetol & 1200 & 1249 & ND & 0,03 \\
\hline 32 & 27,77 & Verbenona & 1211 & 1204 & ND & 0,14 \\
\hline 33 & 31,45 & Bornil acetato & 1289 & 1287 & ND & 0,38 \\
\hline 34 & 28,88 & Timol metil éter & 1234 & 1232 & 0,36 & ND \\
\hline 35 & 29,33 & Carvacrol metil éter & 1244 & 1241 & 0,30 & ND \\
\hline 36 & 31,58 & Timol & 1292 & 1289 & 15,11 & ND \\
\hline 37 & 32,09 & Carvacrol & 1303 & 1298 & 36,48 & ND \\
\hline 38 & 33,77 & $\delta$ - Elemeno & 1341 & 1335 & 0,08 & ND \\
\hline 39 & 37,43 & E-Cariofileno & 1425 & 1417 & 5,03 & 0,48 \\
\hline 40 & 38,86 & $\alpha-$ Humuleno & 1459 & 1452 & 0,24 & ND \\
\hline \multirow[t]{2}{*}{41} & 44,09 & Óxido de cariofileno & 1590 & 1582 & 0,66 & 0,15 \\
\hline & & & & TOTAL & 96,05 & 98,30 \\
\hline
\end{tabular}

ND - Não Detectado.

OS dados da tabela mostram que os constituintes majoritários da L. sidoides são os monoterpenos: cavacrol (36,48\%), $\rho$-cimeno (18,96\%), timol $(15,11 \%)$, $\gamma$-terpineno $(10,25 \%)$, totalizando $80,80 \%$ da composição identificada $(96,16 \%)$ do óleo. Para a amostra comercial os constituintes majoritários encontrados foram os monoterpenos: eucaliptol $(49,14 \%)$, cânfora $(19,27 \%)$, $\alpha$-pineno $(9,72 \%)$ e borneol $(4,98 \%)$ representando $83,11 \%$ do total de componentes identificados $(98,30 \%)$ no óleo. A Figura 1 apresenta os cromatogramas referentes a cada amostra com seus respectivos constituintes majoritários, na qual os picos no cromatograma está associado com os compostos descritos na Tabela 1.

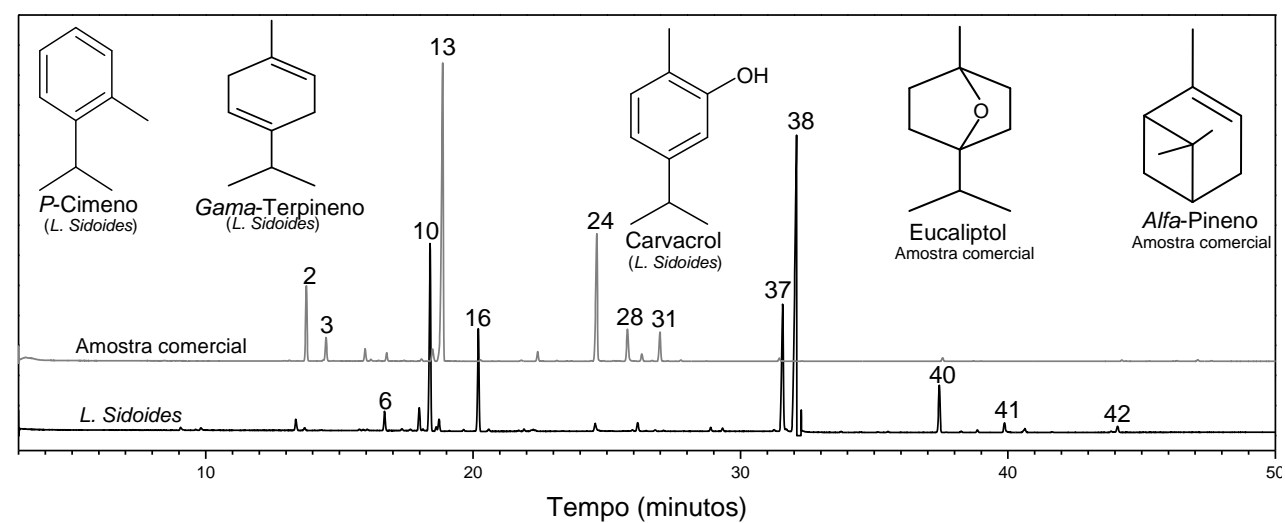

Figura 1. Cromatogramas referentes às análises dos óleos essenciais de L. sidoides e da amostra comercial com seus respectivos constituintes majoritários.

Com base no perfil cromatográfico obtido para a análise da amostra comercial e in natura é possível inferir que se trata de composições químicas significativamente diferentes. A composição química majoritária encontrada na amostra comercial foi comparada com os dados da literatura, para espécies conhecidas popularmente como alecrim, e observa-se semelhança com a relatada por Yosr et al. (2013), na analise do óleo essencial das folhas secas da espécie Rosmarinus 
officinalis L. sendo o eucaliptol (38,40\%), cânfora (16,60\%), borneol (7,30\%) e $\alpha$-pineno $(10,80 \%)$, os constituintes majoritários.

A composição química da $L$. sidoides encontrada neste trabalho converge em parte com a apontada por Cavalcanti et al. (2010) que atribuem a seguinte composição: carvacrol $(46,09 \%), \gamma$ terpineno $(15,49 \%)$ e $\rho$-cimeno $(15,06 \%)$ como constituintes majoritários do óleo essencial das plantas coletadas em regiões distintas do nordeste brasileiro. Já Lima et al. (2011) encontraram o carvacrol (31,68\%), $\rho$-cimeno (19,58\%), 1,8-cineol $(9,26 \%)$ e o $\gamma$-terpineno $(9,21 \%)$ como constituintes majoritários do óleo essencial de Lippia sidoides coletadas em Lavras-MG.

Contudo Cavalcanti et al. (2004) identificaram o timol como um dos componentes majoritários na L. sidoides, correspondendo $80,8 \%$ da sua composição, seguido pelos compostos $\rho$-cimeno (8,6\%), E-cariofileno (5,1\%), v-terpineno (1,6\%), 1,8-cineol $(1,3 \%)$ e $\gamma$-mirceno $(0,9 \%)$. Resultados semelhantes também foram encontrados por Botelho et al. (2007), que identificaram como constituintes majoritários do óleo essencial de plantas, também do nordeste, o timol $(56,7 \%)$ e carvacrol (16,7\%), seguidos dos constituintes minoritários. Fontenelle et al. (2007), analisando o óleo essencial dessa espécie coletada no Estado do Ceará, encontraram o timol $(59,65 \%)$ e o $E$ cariofileno $(10,60 \%)$ como constituintes majoritários.

Com essas análises é possível inferir que a composição química majoritária dos óleos essenciais analisados mostra-se nitidamente diferentes, porém, muitas vezes e, erroneamente, comercializadas com o mesmo nome. Além das duas espécies analisadas neste trabalho, diversas outras são comercializadas com o nome de Alecrim (PLANTAMED, 2014). Desta forma, o presente trabalho corrobora com as análises já descritas na literatura que apontam que a maioria das espécies de plantas medicinais é usada da forma tradicional, sem pesquisas científicas sobre a sua composição ou sua eficácia. Este fato constitui um risco potencial para a população que usa de forma indiscriminada esses recursos (FOGLIO, 2006).

Portanto, é importante que na comercialização destas plantas medicinais seja especificada sua identificação botânica e, preferencialmente, incluindo sua composição Química majoritária, uma vez que as mesmas são bastante utilizadas para o tratamento de enfermidades, principalmente devido ao seu baixo custo e fácil acesso, quando comparadas com medicamentos industrializados.

\section{CONCLUSÕES}

Os óleos essenciais analisados apresentaram composições químicas voláteis consideravelmente diferentes entre si. Sendo que o componente majoritário da amostra in natura (L. sidoides) foi o carvacrol e para a amostra comercial, o eucaliptol. Com base no perfil cromatográfico da amostra de alecrim comercial e com a comparação de dados da literatura foi possível constatar uma grande semelhança com a composição relatada para a $R$. oficcinalis. Portanto, este trabalho ressalta a importância da especificação botânica para reduzir a probabilidade de usos inadequados das plantas medicinais. Uma vez que, como pode-se perceber pelas análises efetuadas, as espécies comercializadas com os mesmos nomes podem possuir constituição química acentuadamente diferentes. Sendo que o efeito pode resultar em ação biológica inesperada e indesejada, pois, as plantas medicinais são cada vez mais utilizadas pela 
população já que apresentam vantagens consideráveis em seu uso, tais como o baixo custo e fácil acesso.

\section{REFERÊNCIAS BIBLIOGRÁFICAS}

1. ADAMS, R. P. Identification of Essential Oil Components by Gas Chromatography/Quadrupole Mass Spectroscopy. Carol Stream: Allured Publishing Corporation, 2001.

2. AL-SEREITIA, M. R.; K M ABU-AMERB \& P SENA. Pharmacology of rosemary (Rosmarinus officinalis Linn.) and its therapeutic potentials. Indian Journal of Experimental Biology v. 37, p.124-131, February 1999.

3. BERTINI, L. M.; PEREIRA, A. F.; OLIVEIRA, C. L. L.; MENEZES, E. A.; MORAIS, S. M.; CUNHA, F. A.; CAVALCANTI, E. S. B. Perfil de sensibilidade de bactérias frente a óleos essenciais de algumas plantas do Nordeste do Brasil. Infarma, v.17, p. 80-83, 2005.

4. CALIXTO, J. B. Efficacy, safety, quality control, marketing and regulatory guidelines for herbal medicines (phytotherapeutic agents). Brazilian Journal of Medical and Biological Research, v. 33, p. 179-189, 2000.

5. CAVALCANTI, E. S. B.; MORAIS, S. M.; LIMA, M. A. A.; SANTANA, E. W. P. Larvicidal activity of essential oils from brazilian plants against Aedes aegypti L. Memórias do Instituto Oswaldo Cruz, v. 99, n. 5, p. 541-544, 2004.

6. CAVALCANTI, S. C. H.; NICULAU, E. S.; BLANK, A. F.; CÂMARA, C. A. G.; ARAÚJO, I. N.; ALVES, P. B. Composition and acaricidal activity of Lippia sidoides essential oil against two-spotted spidermite (Tetranychus urticae Koch). Bioresource Technology, v. 101, p. 829-832, 2010.

7. CRAVEIRO, A. A. Óleos essenciais de plantas do Nordeste. Fortaleza: edições UFC, 1981.

8. DAMIANI, E.; BACCHETTI, T.; PADELLA, L.; TIANO, L.; CARLONI, P. Antioxidant activity of different white teas: Comparison of hot and cold tea infusions. Journal of Food Composition and Analysis, v.33, p. $59-66,2014$.

9. FERREIRA, S. H.; BARATA, L. E. S.; FRANCISCO, S. L. M.; QUEIROZ, S. R. R. Medicamentos a partir de plantas medicinais no Brasil. Academia Brasileira de Ciências, 1998.

10. FOGLIO, M. A.; QUEIROGA, C. L.; SOUSA, I. M. O.; RODRIGUES, R. A. F. Plantas Medicinais como Fonte de Recursos Terapêuticos: Um Modelo Multidisciplinar - Divisão de Fitoquímica, CPQBA/UNICAMP disponível em: <www.multiciencia.unicamp.br/artigos_07/a_04_7.pdf> Acesso em 03 Abril 2014.

11. FONTENELLE, R. O. S.; MORAIS, S. M.; BRITO, E. H. S.; KERNTOPF, M. R.; BRILHANTE, R. S. N.; CORDEIRO,R. A.; TOMÉ, A. R.; QUEIROZ, M. G. R.; NASCIMENTO, N. R. F.; SIDRIM, J. J. C.; ROCHA, M. F. G. Chemical composition, toxicological aspect sandanti fungal activity ofessential oil from Lippia sidoides Cham. J. Antimicrob. Chemother, v. 59, p. 934-940, 2007.

12. HOUGHTON, P.J.. The role of plants in traditional medicine and current therapy. Journal of Alternative and Complementary Medicine I, p.1631-1714, 1995.

13. LEAL-CARDOSO, J. H.; FONTELES, M. C. Pharmacological effects of essential oils of plants of the Northeats of Brazil. In: An. Acad.Bras.Ci., v.71, n.2, 1999.

14. MATOS, F. J. A.; Plantas medicinais: guia de seleção e emprego de plantas usadas em fitoterapia no Nordeste do Brasil, 3ạedição Imprensa Universitária, Fortaleza, Brasil p.394, 
2007.

15. NUNES, G.P.; SILVA, M.F. DA; RESENDE, U.M.; SIQUEIRA, J.M.DE. Plantas medicinais comercializadas por raizeiros no Centro de Campo Grande, Mato Grosso do Sul. Rev. Bras. Farmacogn., v. 13, n. 2, julho-dezembro, 2003.

16. OliVEIRA, M. L. M; BEZERRA, B. M. O; LEITE, L. O.; GIRÃO, C. C.; PINHEIRO, D. C. S.N. Topical continuous use of Lippia sidoides Cham. Essential oil induces cutaneous inflammatory response, but does not delay wound healing process. Journal of Ethnopharmacology, v.15, p.3283-289, 2014.

17. PHEROBASE. Disponível em: <www.pherobase.com> Acesso em 02 de Abril de 2014.

18. PLANTAMED - Plantas e Ervas Medicinais e Fitoterápicos. 2014 Disponível em: <www.plantamed.com.br> Acesso em 29 de março de 2014.

19. SUZUKI, O. Mercado de medicamentos fitoterápicos no Brasil. In: SCHULZ, V.; HÄNSEL, R.; TYLER, V. E. Fitoterapia racional. 4. ed. (1. ed. brasileira). São Paulo: Manole, 2002. Apêndice.

20. VERAS, H. N. H.; RODRIGUES, F. F. G.; COLARES, A. V.; MENEZES, I. R. A.; COUTINHO, H. D. M.; BOTELHO, M. A.; COSTA, J. G. M. Synergistic antibiotic activity of volatile compounds from the essential oil of Lippia sidoides and thymol. Fitoterapia v. 83, p. 508-512, 2012.

21. VIEGAS, M. C.; BASSOLI, D. G. Utilização do índice de retenção linear para caracterização de compostos voláteis em café solúvel utilizando GC-MS e coluna HP-Innowax. Química nova, v. 30, p. 2031-2034, 2007.

22. YOSR, Z.;HNIA, C.; RIM, T.; Mohamed, B. Changes in essential oil composition and phenolic fraction in Rosmarinus officinalis L. var. typicus Batt. organs during growth and incidence on the antioxidant activity. Industrial Crops and Products, v. 43, p. 412-419, 2013. 